\title{
Per-oral endoscopic myotomy (POEM) training and skills evaluation tool: a pilot study
}

\section{다 (1) $\odot$}

Authors

Alexander Schlachterman¹, Ahmed Aziz¹, Bader Alajlan², Samuel Han², Jorge Machicado², Mihir S. Wagh²

Institutions

1 Division of Gastroenterology and Hepatology, Thomas Jefferson University-Philadelphia, Pennsylvania, United States

2 Division of Gastroenterology and Hepatology, University of Colorado-Denver, Colorado, United States

\section{Bibliography}

Endoscopy International Open 2020; 08: E1826-E1831

DOI 10.1055/a-1264-7542

ISSN 2364-3722

(C) 2020. The Author(s).

This is an open access article published by Thieme under the terms of the Creative Commons Attribution-NonDerivative-NonCommercial License, permitting copying and reproduction so long as the original work is given appropriate credit. Contents may not be used for commecial purposes, or adapted, remixed, transformed or built upon. (https://creativecommons.org/licenses/by-nc-nd/4.0/)

\section{Corresponding author}

Mihir S. Wagh, MD, FACG, FASGE, Interventional Endoscopy, Division of Gastroenterology and Hepatology, University of Colorado-Denver, 1635 Aurora Court, F735, Aurora, CO 80045, United States

Fax: +1-720-848-2749

mihir.wagh@cuanschutz.edu

\section{ABSTRACT}

Background and study aims Hands-on training for peroral endoscopic myotomy (POEM) in the United States is limited and without a structured curriculum or assessment tool. Training to competency in POEM is critical and POEM trainees must acquire multiple cognitive and technical skills to achieve proficiency. The aim of this study was to develop a POEM training and skills evaluation tool.

Patients and methods The training protocol included initial explant porcine models followed by live human cases, proctored by a single endoscopist experienced in POEM who prospectively graded trainees for each step ("skill") of the procedure on a 5-point scale. Procedural skills were divided into cognitive and technical skills. Acceptable passing level was considered a score $\geq 4$ for each skill.

Results Three trainees completed a total of 18 cases (8 cases on animal explant models and 10 human cases). Overall, cognitive skills were acquired early in training with scores of $\geq 4$ achieved by $\leq 3$ cases. Technical skills required more cases and direction with scores $\geq 4$ in technical skills achieved by three porcine and eight human cases. Entry of the endoscope into the submucosal space and submucosal tunneling were the most challenging steps followed by myotomy.

Conclusion This pilot study introduces a POEM training and skills evaluation tool for training to competency. Submucosal entry, tunneling, and myotomy were the most challenging to learn while cognitive skills were learned early in training. Evaluation of more trainees at multiple sites will be necessary to further validate the utility of this tool.

\section{Introduction}

Peroral endoscopic myotomy (POEM) has recently emerged as a viable minimally invasive endoscopic approach for the treatment of achalasia and other esophageal motility disorders [1, 2]. Pasricha et al [3] initially described the feasibility of an endoscopic submucosal esophageal myotomy in a pig model in 2007 and the technique was subsequently developed and translated into clinical practice by Inoue [4] in Japan in 2008. The technique has evolved and expanded around the world but hands- on training for POEM in the United States is limited and without a structured curriculum or standardized assessment tool.

There is a significant learning curve for POEM [5-10] and the number of cases required to achieve efficiency and mastery is highly variable [5-11]. There are currently no published training guidelines or established quality metrics for POEM, and POEM training during advanced endoscopy fellowship is limited and varies significantly across programs. Length of procedure along with decreased adverse events (AEs) are frequently referenced by studies when defining the learning curve associated with POEM [5-10]. Furthermore, the learning curve can 
be shortened with mentorship and proctoring [5]. Recently there has been a movement towards competency-based medical education rather than a specific number of procedures to be done during endoscopic training [12]. Training to competency in POEM is critical and POEM trainees must acquire multiple cognitive and technical skills to achieve proficiency. Kishiki and colleagues have proposed a curriculum for pretesting and posttesting of trainees learning POEM [11] but a formal skills assessment tool for evaluating individual steps in performing POEM has not been described.

The aim of this pilot study was to develop a POEM training and skills evaluation tool to evaluate cognitive and technical skills for performing POEM.

\section{Patients and methods}

This pilot study was performed at a tertiary academic medical center in the United States from May 2018 to November 2018. The study was approved by the Institutional Review Board (IRB) at the University of Colorado. The aim of this study was to develop a POEM training and skills evaluation tool.

Trainees included interventional endoscopy fellows with no prior hands-on experience in endoscopic submucosal dissection (ESD) or POEM, and an interventional endoscopist (1 year on university faculty after completion of an advanced endoscopy fellowship). All participants were trained in interventional endoscopy during their 4th year advanced endoscopy fellowship. As part of their advanced endoscopy fellowship, each trainee had performed complex polypectomy, endoscopic mucosal resection, luminal stenting, > 250 endoscopic ultrasound cases and $>300$ endoscopic retrograde cholangiopancreatographies (ERCPs). Before the study, trainees had listened to lectures on POEM and ESD as part of their curriculum and attended endoscopy conferences where third space endoscopy (TSE) concepts and techniques were discussed by senior experienced endoscopists. All trainees had observed (but not performed) ESD and POEM cases during their fellowship, and the faculty endoscopist who had completed his advanced endoscopy fellowship 1 year prior to the study had observed ESD cases performed by Japanese experts and performed $<25$ ESD cases.

The training protocol included performing POEM initially on explant porcine models followed by live human cases, proc- tored by a single endoscopist (MSW) experienced (>150 POEM procedures) in POEM. Skills required for performing POEM were divided into two groups: (1) cognitive skills and (2) technical skills ( Table $\mathbf{1}$ ).

Cognitive skills included: (1) diagnostic endoscopic evaluation of the gastroesophageal (GE) junction and stomach; (2) appropriate site selection for mucosal incision; (3) identification of esophageal wall layers; (4) identification of planes and orientation during submucosal tunneling; (5) identification of anatomical planes and structures at GE junction and cardia; and (6) identification of circular and longitudinal muscle planes. Technical skills included: (1) entry of the endoscope into the submucosal space; (2) submucosal tunneling; (3) performance of myotomy; (4) management of bleeding; (5) managing of mucosal injury or perforation; and (6) incision closure.

Trainees were graded for each of the cognitive and technical skills on a 5-point scale as follows: 1 = trainer had to take over, 2 =trainer provided technical assistance, but trainee was able to complete, 3 =trainer provided substantial verbal guidance, $4=$ trainer provided minimal verbal guidance, and $5=$ trainee performed completely independently (Supplemental table).

\section{Definitions}

Acceptable passing level for each skill was considered at skill level $\geq 4$ for that skill. AEs were recorded per published American Society for Gastrointestinal Endoscopy criteria [13].

\section{Ex-vivo POEM procedures}

All ex-vivo training was performed in explant porcine models comprising of esophagus, stomach and duodenum assembled on a training tray. Technical steps of POEM were as described below for human POEM cases.

\section{Human POEM procedures}

All patients had pre-procedure diagnostic upper endoscopy, barium esophagram and high-resolution esophageal manometry, to confirm the diagnosis and rule out alternate and coexisting conditions. Patients were asked to stay on a liquid diet for 2 days before the procedure to allow adequate esophageal clearance for visualization during POEM and to minimize risk of AEs.

POEM was performed in the supine position in the endoscopy unit under general anesthesia with endotracheal intuba-

$\checkmark$ Table 1 Cognitive and technical skills for POEM.

\begin{tabular}{|l|l|}
\hline Cognitive skills & Technical skills \\
\hline 1. Diagnostic endoscopic evaluation of GE junction and stomach & 1. Submucosal entry \\
\hline 2. Appropriate site selection for mucosal incision & 2. Submucosal tunneling \\
\hline 3. Identification of esophageal wall layers & 3. Performance of myotomy \\
\hline 4. Identification of planes and orientation during submucosal tunneling & 4. Management of bleeding (hemostasis) \\
\hline 5. Identification of anatomical planes and structures at GE junction and cardia & 5. Management of mucosal injury or perforation \\
\hline 6. Identification of circular and longitudinal muscle planes & 6. Incision closure \\
\hline POEM, per-oral endoscopic myotomy; GE, gastroesophageal. & \\
\hline
\end{tabular}




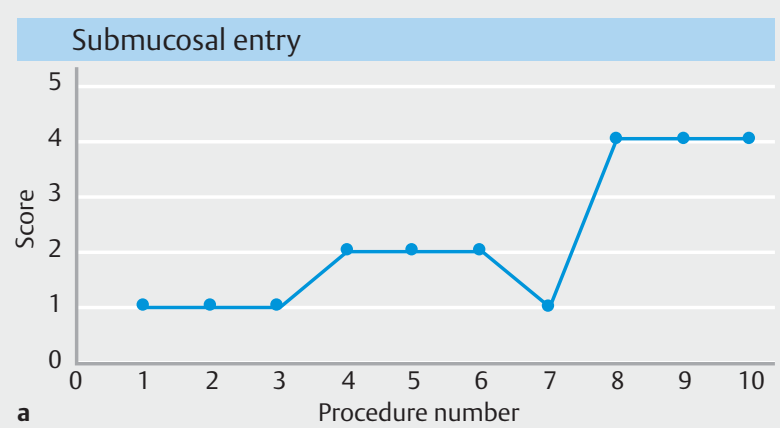

a

\section{Submucosal tunneling}

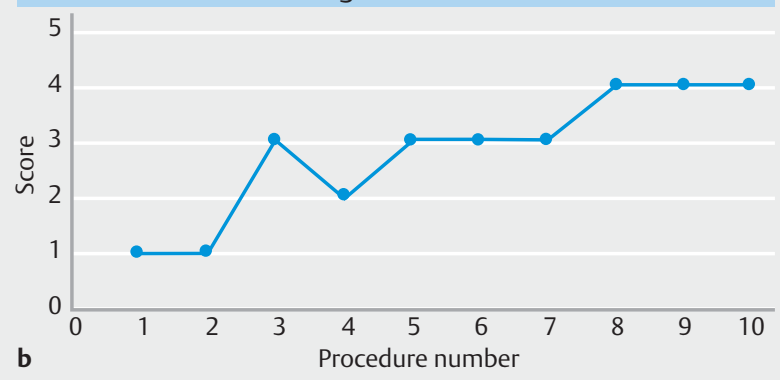

Myotomy

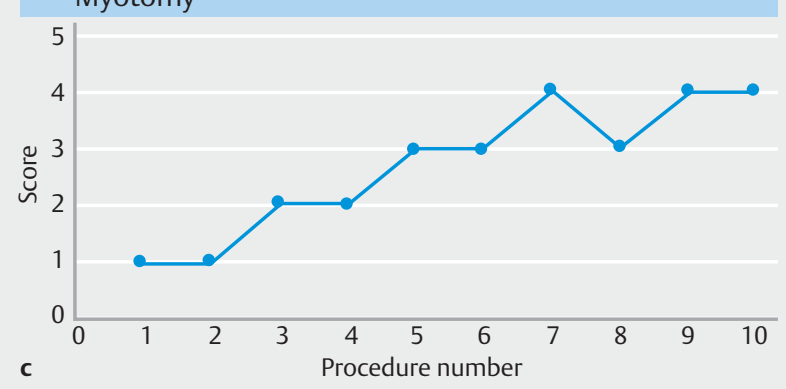

c Procedure number

- Fig. 1 Skills assessment during a submucosal entry, b submucosal tunneling, and $\mathbf{c}$ myotomy during human POEM procedures.

tion. Antibiotics were administered peri-procedurally, typically a semi-synthetic penicillin with beta-lactamase inhibitor (ampicillin-sulbactam), or a fluoroquinolone and metronidazole (if penicillin allergy). Technical steps of POEM were as previously described $[4,14]$. A posterior submucosal tunnel with a posterior myotomy in the 5 o'clock position was performed with the T-type Hybrid knife (ERBE USA, Marietta, GA). Bleeding during the procedure was treated with the dissection knife and/or Coagrasper (Olympus America, Center Valley, Pennsylvania, United States). The mucosal incision was closed with endoscopic clips (Quick Clip Pro, Olympus America, Center Valley, Pennsylvania, United States or Resolution 360 clips, Boston Scientific, Marlborough, Massachusetts, United States).

All patients were admitted for overnight observation and an esophagram was obtained the following morning to exclude leak after POEM. Patients were discharged on a liquid diet and advanced to soft foods in 1 week. Oral antibiotics were continued for 7 days. Patients were seen for clinic follow-up and surveillance upper endoscopy.

\section{Results}

Trainees completed a total of 18 procedures ( 8 cases on animal explant models and 10 human cases) and were evaluated by one experienced interventional endoscopist experienced in POEM. Mean age of patients was 54.1 years (range 25-68 years) and $50 \%$ were male. Indications for POEM included achalasia type 1 (10\%), type $2(50 \%)$, type $3(20 \%)$ and esophagogastric junction outflow obstruction (EGJOO) (20\%). Mean submucosal tunnel length was $14 \mathrm{~cm}$ (range 12-17) and mean myotomy length was $11.8 \mathrm{~cm}$ (range 8-16). Mean Eckardt score was 6.9 (range $4-10$ ) on presentation and decreased to mean score 0.88 (range $0-2$ ) after POEM. No AEs were seen after POEM. One patient with EGJOO had persistent hiccups after POEM but had significant improvement in Eckardt score to 1 (with no dysphagia).

\section{Cognitive skills assessment}

All trainees were able to perform diagnostic endoscopic evaluation of the GE junction and stomach with a score of $\geq 4$ on their first porcine and first human procedure. All trainees were able to select an appropriate site for mucosal incision with a score of $\geq 4$ on their first porcine and third human POEM case. Trainees required a mean of 1.67 procedures (range $1-3$ ) in porcine models and 1 human POEM procedure to achieve a score of $\geq 4$ for identification of esophageal wall layers. Trainees required a mean of 1.67 procedures (range $1-3$ ) in porcine models and three human POEM procedures to achieve a score of $\geq 4$ for identification of planes and orientation during submucosal tunneling, and for identification of anatomical planes and structures at the GE junction and cardia. Trainees required a mean of two procedures (range 1-3) in porcine models and one human POEM procedure to achieve a score of $\geq 4$ for identification of circular and longitudinal muscle planes.

\section{Technical skills assessment}

Trainees achieved a score of $\geq 4$ at a mean of 2.67 porcine procedures (range $2-3$ ) and eight human procedures for submucosal entry, and also for submucosal tunneling, suggesting that these two steps were the most challenging steps. Myotomy was also one of the more challenging steps of the procedure. Trainees required a mean of two procedures (range $1-3$ ) in porcine models and seven human procedures to achieve a score of $\geq 4$ for myotomy. Management of bleeding could only be assessed in human cases and score $\geq 4$ was achieved at the fourth human case where bleeding was encountered. There were no mucosal perforations in either porcine or human cases in this study. All trainees achieved a score $\geq 4$ for mucosal incision closure with their first procedure in both porcine and human cases. Esophagram obtained the day after human POEM confirmed no leak in all human POEM cases.

Cognitive skills were acquired early in training with scores of $\geq 4$ achieved by $\leq 3$ cases. Technical skills required more cases and direction with scores $\geq 4$ in technical skills achieved by three porcine and eight human cases. Entry of the endoscope into the submucosal space and submucosal tunneling were the most challenging steps followed by myotomy ( $\triangleright$ Fig. 1). 


\section{Discussion}

TSE including ESD and POEM is a new addition to the endoscopic spectrum. Training in these newer procedures such as POEM requires additional skills with a steep and variable learning curve [5-10]. While there are advanced endoscopy training programs and established curricula for ERCP and EUS [15-17] there is currently no standard curriculum for training in POEM. A proposed strategy for training in these newer advanced endoscopic procedures such as ESD or POEM involves observation of live procedures performed by experts, and then performing initial procedures on animal models, followed by first human cases proctored by an experienced endoscopist proficient in that specific procedure such as POEM [18]. However, it should be noted that these suggestions for training are opinions based on individual endoscopist experiences and not validated in studies evaluating POEM skills.

With the move to competency-based endoscopy training, evaluation of skills required for performing POEM is crucial. We have created a POEM training and skills evaluation tool and introduce it in this pilot study. The steps involved in the POEM procedure were broken down into cognitive and technical skills and each skill was graded by the proctoring endoscopist.

The technical challenge in entering the human submucosal space may be explained due to limited submucosal dissection performed by the trainee due to concern for muscle perforation after mucosal incision, hesitation by the trainee endoscopist in extending the mucosotomy for entry, and management of mucosal and submucosal bleeding during entry in human patients. Similarly, submucosal tunneling and myotomy were technically challenging as well, likely due to difficulty in learning submucosal dissection while preserving the overlying mucosa, management of intervening blood vessels during tunneling, and concern for injury to deeper extra-esophageal structures during muscle incision.

The highlights of our study include stepwise evaluation of the entire POEM procedure by deconstructing it into assessible individual cognitive and technical components. This type of mentored evaluation and grading with a standardized tool may allow formal training to achieve competence. This tool can be used by trainers to evaluate trainees for competency in the various steps of the POEM procedure and will be essential to institutions establishing their TSE programs.

We also acknowledge the limitations of our study. The main limitation is that the study was performed at a single institution with a small number of trainees, limited number of procedures, and with a single instructor. Hence our results are likely not generalizable to all trainees and trainers, across different sites and experience levels. Second, other cognitive aspects were not evaluated such as knowledge of accessories/knives, electrosurgery settings, and injection solutions, often considered pre-requisites for POEM training. Third, management of AEs was not adequately assessed in this small study because overall AEs are rare and because bleeding could only be assessed in human cases. Similarly, management of mucosal perforations was not assessed. In addition, human cases did not include difficult anatomy such as sigmoid esophagus/end-stage achalasia. Thus, results from this study may not be applicable to all POEM scenarios, demonstrating potential selection bias. However, we believe this study introduces the important concept of training for POEM to competency, with objective assessment of every step in the procedure. Finally, we accept that the small number of trainees and procedures in this pilot study makes assessing competency difficult, but it should be emphasized that the goal of this study was to introduce a POEM training and skills evaluation tool, rather than setting or defining competency standards. We hope that this tool will lay the groundwork for future studies to assess POEM training and competency.

\section{Conclusion}

This pilot study introduces a POEM training and skills evaluation tool. Our study shows that submucosal entry, tunneling, and myotomy were the most challenging to learn while cognitive skills were learned early in training. Evaluation of POEM procedures performed by more trainees at multiple sites will be necessary to further validate the utility of this tool.

\section{Competing interests}

Dr. Schlachterman is a consultant for Medtronic, Lumendi and ConMed. Dr. Wagh is a consultant for Boston Scientific, Olympus, Medtronic, and Incyte.

\section{References}

[1] Werner YB, Hakanson B, Martinek J et al. Endoscopic or surgical myotomy in patients with idiopathic achalasia. N Engl J Med 2019; 381: 2219-2229

[2] Khashab MA, Vela MF, Thosani $\mathrm{N}$ et al. ASGE guideline on the management of achalasia. Gastrointest Endosc 2020; 91: 213-227

[3] Pasricha PJ, Hawari R, Ahmed I et al. Submucosal endoscopic esophageal myotomy: a novel experimental approach for the treatment of achalasia. Endoscopy 2007; 39: 761-764

[4] Inoue H, Minami H, Kobayashi Y et al. Peroral endoscopic myotomy (POEM) for esophageal achalasia. Endoscopy 2010; 42: 265-271

[5] Kurian AA, Dunst CM, Sharata A et al. Peroral endoscopic esophageal myotomy: defining the learning curve. Gastrointest Endosc 2013; 77: 719-725

[6] Teitelbaum EN, Soper NJ, Arafat FO et al. Analysis of a learning curve and predictors of intraoperative difficulty for peroral esophageal myotomy (POEM). J Gastrointest Surg 2014; 18: 92-99

[7] Patel KS, Calixte R, Modayil R] et al. The light at the end of the tunnel: a single-operator learning curve analysis for per oral endoscopic myotomy. Gastrointest Endosc 2015; 81: 1181-1187

[8] El Zein M, Kumbhari V, Ngamruengphong $S$ et al. Learning curve for peroral endoscopic myotomy. Endosc Int Open 2016; 4: E577-E582

[9] Lv H, Zhao N, Zheng Z et al. Analysis of the learning curve for peroral endoscopic myotomy for esophageal achalasia: single-center, twooperator experience. Dig Endosc 2017; 29: 299-306

[10] Liu Z, Zhang X, Zhang W et al. Comprehensive evaluation of the learning curve for peroral endoscopic myotomy. Clin Gastroenterol Hepatol 2018; 16: 1420-1426 
[11] Kishiki T, Lapin B, Wang C et al. Teaching peroral endoscopic myotomy (POEM) to surgeons in practice: an "into the fire" pre/post-test curriculum. Surg Endosc 2018; 32: 1414-1421

[12] Faulx AL, Lightdale JR. ASGE Standard of Practice Committee. et al. Guidelines for privileging, credentialing, and proctoring to perform GI endoscopy. Gastrointest Endosc 2017; 85: 273-281

[13] Cotton PB, Eisen GM, Aabakken L et al. A lexicon for endoscopic adverse events: report of an ASGE workshop. Gastrointest Endosc 2010; 71: $446-454$

[14] Mittal C, Wagh MS. Technical advances in per-oral endoscopic myotomy (POEM). Am J Gastroenterol 2017; 112: 1627-1631
[15] Jorgensen J, Kubiliun N. ASGE Training Committee. et al. Endoscopic retrograde cholangiopancreatography (ERCP): core curriculum. Gastrointest Endosc 2016; 83: 279-289

[16] Cassani L, Aihara H, Anand GS et al. Core curriculum for EUS. Gastrointest Endosc 2020; 92 (3): 469-473

[17] Wani S, Han S, Simon V et al. Setting minimum standards for training in EUS and ERCP: results from a prospective multicenter study evaluating learning curves and competence among advanced endoscopy trainees. Gastrointest Endosc 2019; 89: 1160-1168.e9

[18] Eleftheriadis $\mathrm{N}$, Inoue $\mathrm{H}$, Ikeda $\mathrm{H}$ et al. Training in peroral endoscopic myotomy (POEM) for esophageal achalasia. Therapeutics and clinical risk management 2012; 8: 329-342 


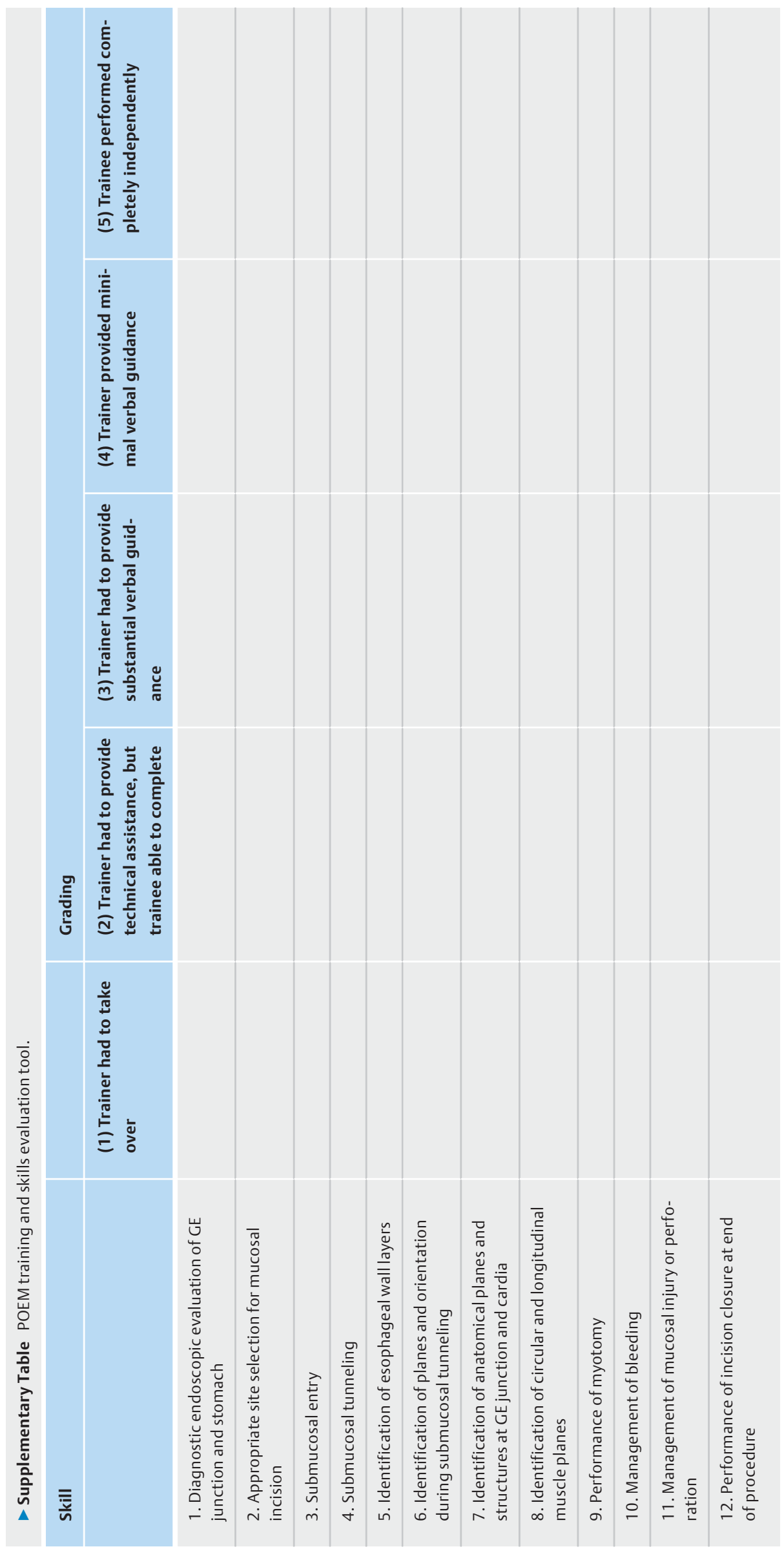

